\title{
Geometry on the lines of polar spine spaces
}

\author{
Krzysztof PetelczyciD, KRzysztof Prażmowski®, And \\ MARIUSZ ŻYNEL(D
}

\begin{abstract}
The concept of spine geometry over a polar Grassmann space is introduced. We determine conditions under which the structure of lines together with a binary coplanarity relation is a sufficient system of primitive notions for polar spine spaces. These assumptions also allow to characterize polar spine spaces in terms of the binary relation of being in one pencil of lines.
\end{abstract}

Mathematics Subject Classification. 51A15, 51A45.

Keywords. Grassmann space, Projective space, Polar space, Spine space, Coplanarity, Pencil of lines.

\section{Introduction}

In $[7,8]$ we proved that lines with coplanarity relation $\boldsymbol{\pi}$ suffice to define polar Grassmann spaces (cf. [5,6,9]) and spine spaces (cf. [10,12,13,15]). A natural question arises: can we do the same for a geometry that possesses both 'polar' and 'spine' properties?

Although, regardless of this question, a polar spine geometry $\mathfrak{M}$ is worth considering in details, this is not addressed in the paper. We determine only those properties that are necessary for the proof of our main theorem. Firstly, we have to establish parameters for which $\mathfrak{M}$ is nondegenerate. Following $[7,8]$ we would like to identify points of our geometry with vertices of bundles of lines. Every bundle is covered by semibundles of lines, each contained in some maximal strong subspace of $\mathfrak{M}$. So, maximal strong subspaces are essential here and we need to know what they and their intersections look like. Generally, as in all 'Grassmann-type' spaces, in $\mathfrak{M}$ we have stars and tops. Stars and tops can be either projective or semiaffine (cf. [16]) spaces, so actually the family of all maximal strong subspaces splits into four classes. In this $\mathfrak{M}$ resembles a 
spine space. However, in spine spaces and polar spaces all maximal subspaces belonging to the same class have equal dimensions, which is not true for $\mathfrak{M}$ : the dimension of projective stars is not constant. The next and main difference between $\mathfrak{M}$ and spine or polar spaces is that $\mathfrak{M}$ can be disconnected. We do not solve the problem of connectedness of $\mathfrak{M}$ entirely, but we shed some light on this.

Let us return to the main question. The maximal cliques of coplanarity in $\mathfrak{M}$ are the same as in spine spaces and quite a bit of the reasoning from [8] can be repeated. There are some new difficulties though, mainly caused by multidimensional stars. It is hard to use techniques from [8] to characterize the family of all maximal $\boldsymbol{\pi}$-cliques in terms of lines and the relation $\boldsymbol{\pi}$. Nevertheless, all the proper top semibundles are just what we need in the rest of our reasoning. So, it suffices to distinguish them in the set of ('uniformly') definable maximal $\boldsymbol{\pi}$-cliques. Since the dimension of top semibundles might be the same as the dimension of some star semibundles a couple of additional assumptions must be imposed on $\mathfrak{M}$ (cf. Theorem 4.5).

Pencils of lines are an important tool to recognize and compare the dimensions of maximal $\boldsymbol{\pi}$-cliques. We consider line-pencil geometry on these cliques to do this. The same can be done on the whole polar spine space $\mathfrak{M}$. Then the relation $\boldsymbol{\rho}$ of being in one pencil is simply the binary collinearity relation. In the last section we show that top semibundles as well as proper pencils can be characterized in terms of $\boldsymbol{\rho}$. Consequently the set of lines equipped with the relation $\rho$ is a sufficient system of primitive notions for both point-line and line-pencil geometries on $\mathfrak{M}$. We prove even more: all these structures are definitionally equivalent, i.e. they all are mutually definable (cf. Theorem 5.2). We say that 'something is definable' when it can be characterized in the language of first-order logic (cf. [17, Chapter 4.6]).

\section{Generalities}

A point-line structure $\mathfrak{A}=\langle S, \mathcal{L}\rangle$, where the elements of $S$ are called points, the elements of $\mathcal{L}$ are called lines, and where $\mathcal{L} \subset 2^{S}$, is said to be a partial linear space, or a point-line space, if two distinct lines share at most one point and every line is of size (cardinality) at least 2 (cf. [2]).

A subspace of $\mathfrak{A}$ is any set $X \subseteq S$ with the property that every line which shares two or more points with $X$ is entirely contained in $X$. We say that a subspace $X$ of $\mathfrak{A}$ is strong if any two points in $X$ are collinear. A plane in $\mathfrak{A}$ is a strong subspace $E$ of $\mathfrak{A}$ with the property that the restriction of $\mathfrak{A}$ to $E$ is a projective plane. If $S$ is strong, then $\mathfrak{A}$ is said to be a linear space.

Let us fix a nonempty subset $\mathcal{D} \subset S$ and consider the set

$$
\mathcal{L}_{\mathcal{D}}:=\{k \cap \mathcal{D}: k \in \mathcal{L} \text { and }|k \cap \mathcal{D}| \geq 2\} .
$$


The structure

$$
\mathfrak{M}:=\left\langle\mathcal{D}, \mathcal{L}_{\mathcal{D}}\right\rangle
$$

is a fragment of $\mathfrak{A}$ induced by $\mathcal{D}$ and it is a partial linear space itself. The incidence relation in $\mathfrak{M}$ is again $\epsilon$, inherited from $\mathfrak{A}$, but limited to the new pointset and lineset. Since $\mathcal{D}$ is arbitrary we assume that

$$
|L \cap \mathcal{D}| \leq 1 \quad \text { or } \quad|L \backslash \mathcal{D}| \leq 1 \quad \text { for all } L \in \mathcal{L}
$$

to give the following definitions their natural geometrical sense. Following a standard convention we call the points of $\mathfrak{M}$ proper, and the points in $S \backslash \mathcal{D}$ improper. The set $S \backslash \mathcal{D}$ will be called the horizon of $\mathfrak{M}$. To every line $L \in \mathcal{L}_{\mathcal{D}}$ we can uniquely assign the line $\bar{L} \in \mathcal{L}$, the closure of $L$, such that $L \subseteq \bar{L}$. For a subspace $X \subseteq \mathcal{D}$ the closure of $X$ is the minimal subspace $\bar{X}$ of $\mathfrak{A}$ containing $X$. A line $L \in \mathcal{L}_{\mathcal{D}}$ is said to be a projective line if $L=\bar{L}$, and it is said to be an affine line if $|\bar{L} \backslash L|=1$. We write $\mathcal{A}$ for the class of affine lines. In case $\mathcal{L}_{\mathcal{D}}$ contains projective or affine lines only, then $\mathfrak{M}$ is a semiaffine geometry (for details on terminology and axiom systems see [16]). In this approach an affine space is a particular case of a semiaffine space. For affine lines $L_{1}, L_{2} \in \mathcal{L}_{\mathcal{D}}$ we can define parallelism in a natural way:

$$
L_{1}, L_{2} \text { are parallel iff } \overline{L_{1}} \cap \overline{L_{2}} \cap(S \backslash \mathcal{D}) \neq \emptyset .
$$

We say that $E$ is a plane in $\mathfrak{M}$ if $\bar{E}$ is a plane in $\mathfrak{A}$. Observe that there are two types of planes in $\mathfrak{M}$ : projective and semiaffine. A semiaffine plane $E$ arises from $\bar{E}$ by removing a point or a line. As a result we get a punctured plane or an affine plane, respectively. For lines $L_{1}, L_{2} \in \mathcal{L}_{\mathcal{D}}$ we say that they are coplanar and write

$$
L_{1} \pi L_{2} \text { iff there is a plane } E \text { such that } L_{1}, L_{2} \subset E \text {. }
$$

Let $E$ be a plane in $\mathfrak{M}$ and $U \in \bar{E}$. The set

$$
\mathbf{p}(U, E):=\left\{L \in \mathcal{L}_{\mathcal{D}}: U \in \bar{L} \subset \bar{E}\right\}
$$

will be called a proper pencil if $U$ is a proper point, or a parallel pencil otherwise. The point $U$ is said to be the vertex and the plane $E$ is said to be the base plane of that pencil. We write

$$
L_{1} \rho L_{2} \text { iff there is a pencil } p \text { such that } L_{1}, L_{2} \in p .
$$

Recall that for any binary relation $\varrho$ a set is said to be a $\varrho$-clique iff every two elements of this set are $\varrho$-related. If $L_{1} \rho L_{2}$, then clearly $L_{1} \boldsymbol{\pi} L_{2}$. This means that every $\boldsymbol{\rho}$-clique is a $\boldsymbol{\pi}$-clique.

For lines $L_{1}, L_{2}, \ldots, L_{n}$ and a binary relation $\boldsymbol{\delta}$ on lines in $\mathfrak{M}$ we introduce

$$
\begin{aligned}
& \boldsymbol{\Delta}_{\boldsymbol{\delta}}^{n}\left(L_{1}, L_{2}, \ldots, L_{n}\right) \text { iff } \\
& \quad \neq\left(L_{1}, L_{2}, \ldots, L_{n}\right) \text { and } L_{i} \boldsymbol{\delta} L_{j} \text { for all } i, j=1, \ldots, n, \\
& \quad \text { and for all } M_{1}, M_{2} \in \mathcal{L} \text { if } M_{1}, M_{2} \delta L_{1}, L_{2}, \ldots, L_{n}, \text { then } M_{1} \delta M_{2},
\end{aligned}
$$


where $\neq(\ldots)$ means pairwise distinct. Actually, only the case $n=3$ is exploited in our paper so, we write $\boldsymbol{\Delta}_{\delta}$ instead of $\boldsymbol{\Delta}_{\delta}^{3}$. However, for instance in polar Grassmann spaces the minimal number $n$ of lines for which $\boldsymbol{\Delta}_{\pi}^{n}$ can be true is the dimension of stars (cf. $[7,8])$. Let

$$
\left[L_{1}, L_{2}, \ldots, L_{n}\right]_{\delta}:=\left\{L \in \mathcal{L}: L \delta L_{1}, L_{2}, \ldots, L_{n}\right\} .
$$

If $\boldsymbol{\Delta}_{\boldsymbol{\delta}}^{n}\left(L_{1}, L_{2}, \ldots, L_{n}\right)$ and $\boldsymbol{\delta}$ is reflexive, then $\left[L_{1}, L_{2}, \ldots, L_{n}\right]_{\delta}$ is a unique maximal $\boldsymbol{\delta}$-clique spanned by the lines $L_{1}, L_{2}, \ldots, L_{n}$. Finally, for $n \geq 3$

$$
\mathcal{K}_{\delta}^{n}:=\left\{\left[L_{1}, L_{2}, \ldots, L_{n}\right]_{\delta}: L_{1}, L_{2}, \ldots, L_{n} \in \mathcal{L} \text { and } \boldsymbol{\Delta}_{\delta}^{n}\left(L_{1}, L_{2}, \ldots, L_{n}\right)\right\} .
$$

For a subspace $X$ of $\mathfrak{M}$ and $U \in \bar{X}$ we write

$$
\mathrm{L}(X)=\left\{L \in \mathcal{L}_{\mathcal{D}}: L \subset X\right\}, \quad \mathrm{L}_{U}(X)=\left\{L \in \mathcal{L}_{\mathcal{D}}: U \in \bar{L} \text { and } L \subseteq X\right\} .
$$

If $E$ is a plane in $\mathfrak{M}$, then the set $\mathrm{L}(E)$ will be called a flat. If $X$ is a strong subspace of $\mathfrak{M}$, then $\mathrm{L}_{U}(X)$ is said to be a semibundle. As the vertex $U$ of a semibundle $\mathrm{L}_{U}(X)$ can be proper or improper, we call the semibundle proper or improper accordingly. We omit the adjective when we mean a semibundle in general. The subspace $E$ as above will be called the base of the flat $\mathrm{L}(E)$ and the subspace $X$ will be called the base of the semibundle $\mathrm{L}_{U}(X)$. We say that three lines form a tripod if they meet in a point, are pairwise coplanar, and are not contained in a plane.

\subsection{Grassmann spaces, polar Grassmann spaces, and spine spaces}

Polar spine spaces, which are introduced in the next subsection, are connected to Grassmann spaces, polar Grassmann spaces and spine spaces. Let us briefly recall definitions of these geometries.

Let $V$ be a vector space of dimension $n$ with $3 \leq n<\infty$. The set of all subspaces of $V$ will be written as $\operatorname{Sub}(V)$ and the set of all $k$-dimensional subspaces (or $k$-subspaces in short) as $\operatorname{Sub}_{k}(V)$. By a $k$-pencil we mean the set of the form

$$
\mathbf{p}(H, B):=\left\{U \in \operatorname{Sub}_{k}(V): H \subset U \subset B\right\}=[H, B]_{k},
$$

where $H \in \operatorname{Sub}_{k-1}(V), B \in \operatorname{Sub}_{k+1}(V)$, and $H \subset B$. The family of all such $k$-pencils will be denoted by $\mathcal{P}_{k}(V)$. A Grassmann space (also known as a space of pencils or a projective Grassmannian) is a point-line space

$$
\mathbf{P}_{k}(V)=\left\langle\operatorname{Sub}_{k}(V), \mathcal{P}_{k}(V)\right\rangle,
$$


with $k$-subspaces of $V$ as points and $k$-pencils as lines. It is known that there are two classes of maximal strong subspaces in $\mathbf{P}_{k}(V)$ : stars of the form

$$
\mathrm{S}(H)=\left\{U \in \operatorname{Sub}_{k}(V): H \subset U\right\},
$$

where $H \in \operatorname{Sub}_{k-1}(V)$, and tops of the form

$$
\mathrm{T}(B)=\left\{U \in \operatorname{Sub}_{k}(V): U \subset B\right\},
$$

where $B \in \operatorname{Sub}_{k+1}(V)$. For more details concerning properties of Grassmann spaces see $[5,6,18]$.

A spine space is a fragment of a Grassmann space chosen as follows. Let $W$ be a fixed subspace of $V, \operatorname{dim}(W)=w$, and let $m$ be an integer with

$$
k-n+w \leq m \leq k, w .
$$

From the points of the Grassmann space $\mathbf{P}_{k}(V)$ we take those which as subspaces of $V$ meet $W$ in dimension $m$, that is

$$
\mathcal{F}_{k, m}(W):=\left\{U \in \operatorname{Sub}_{k}(V): \operatorname{dim}(U \cap W)=m\right\} .
$$

As new lines we take those lines of $\mathbf{P}_{k}(V)$ which have at least two new points so, applying (1) we have

$$
\mathcal{G}_{k, m}(W):=\mathcal{P}_{k}(V)_{\mathcal{F}_{k, m}(W)} .
$$

The point-line structure

$$
\mathbf{A}_{k, m}(V, W):=\left\langle\mathcal{F}_{k, m}(W), \mathcal{G}_{k, m}(W)\right\rangle
$$

will be called a spine space. This concept was coined in [10] and developed in [12-15]. The classification of maximal strong subspaces in $\mathbf{A}_{k, m}(V, W)$ is shown in Table 2.

Let $\xi$ be a nondegenerate reflexive sesquilinear form of index $r$ on $V$. For $U, W \in \operatorname{Sub}(V)$ we write $U \perp W$ iff $\xi(U, W)=0$, meaning that $\xi(u, w)=0$ for all $u \in U, w \in W$. Then the set of all totally isotropic subspaces of $V$ is

$$
\mathrm{Q}:=\{U \in \operatorname{Sub}(V): U \perp U\},
$$

and $\mathrm{Q}_{k}:=\mathrm{Q} \cap \operatorname{Sub}_{k}(V)$. The set $\mathrm{Q}_{k}$ is nonempty iff

$$
k \leq r .
$$

The incidence structure $\left\langle\mathrm{Q}_{1}, \mathrm{Q}_{2}\right\rangle$ is a polar space. The structure

$$
\mathbf{P}_{k}(\mathrm{Q}):=\left\langle\mathrm{Q}_{k}, \mathcal{P}_{k}(\mathrm{Q})\right\rangle,
$$

with pencils of totally isotropic $k$-subspaces as lines is a polar Grassmann space.

Tops in polar Grassmann spaces are of the same form as in projective Grassmann spaces, i.e. $[\Theta, B]_{k}$ where $B \in \mathrm{Q}_{k+1}$, but stars are $k$-intervals $[H, Y]_{k}$, where $H \in \mathrm{Q}_{k-1}, Y \in \mathrm{Q}_{r}$, and $H \subseteq Y$. So, it is seen that in both cases, up to an isomorphism, they are projective spaces and as such tops are of dimension $k$ while stars are of dimension $r-k$. 
Fact 1.1. Two distinct lines $L_{i}=\mathbf{p}\left(H_{i}, B_{i}\right), i=1,2$ are coplanar in $\mathbf{P}_{k}(\mathrm{Q})$ iff they share some point $U$ and

(i) either $H_{1}=H_{2}, B_{1} \perp B_{2}$ and $U=B_{1} \cap B_{2}$,

(ii) or $B_{1}=B_{2}$ and $U=H_{1}+H_{2}$.

\subsection{Polar spine spaces}

From the points of the polar Grassmann space $\mathbf{P}_{k}(\mathrm{Q})$ we take those which as subspaces of $V$ meet $W$ in dimension $m$, that is:

$$
\mathcal{F}_{k, m}(\mathrm{Q}, W):=\left\{U \in \mathrm{Q}_{k}: \operatorname{dim}(U \cap W)=m\right\} .
$$

Using (1) we introduce

$$
\mathcal{G}_{k, m}(\mathrm{Q}, W):=\mathcal{P}_{k}(\mathrm{Q})_{\mathcal{F}_{k, m}(\mathrm{Q}, W)} .
$$

The point-line structure:

$$
\mathfrak{M}=\mathbf{A}_{k, m}(\mathrm{Q}, W):=\left\langle\mathcal{F}_{k, m}(\mathrm{Q}, W), \mathcal{G}_{k, m}(\mathrm{Q}, W)\right\rangle
$$

will be called a polar spine space.

Let $r_{W}=\operatorname{ind}(\xi \mid W)$ be the index of the form $\xi$ restricted to $W$. If $r_{W}<m$, then there is no totally isotropic subspace of $V$ which meets $W$ in some $m$ dimensional subspace. Every $U \in \mathrm{Q}$ can be extended to $Y \in \mathrm{Q}_{r}$. Assume that $\operatorname{dim}(Y \cap W)>r-k+m$ for all $Y \in \mathrm{Q}_{r}$. This means that all totally isotropic subspaces of $V$ which meet $W$ in some $m$-dimensional subspace, are at most $(k-1)$-dimensional. On the other hand, this assumption implies $r_{W}>r-k+m$. Thus

$$
m \leq r_{W} \leq r-k+m
$$

is a sufficient condition for $\mathcal{F}_{k, m}(\mathrm{Q}, W) \neq \emptyset$.

Now, let us have a look at the structure of strong subspaces of $\mathfrak{M}$. For the same reason as in the case of spine spaces, maximal strong subspaces are called: $\alpha$-stars, $\omega$-stars, $\alpha$-tops and $\omega$-tops (cf. Tables 1 and 2). The main difference is that $\alpha$-stars in $\mathfrak{M}$ are somewhat smaller, like stars in polar Grassmann spaces in contrast to stars in Grassmann spaces, and the dimension of an $\omega$ star depends on the intersection of the surrounding maximal totally isotropic subspace with $W$. Note also here that a strong subspace of $\mathfrak{M}$ is a slit space, i.e. a projective space with one of its subspaces removed (cf. $[3,16])$. For details see Table 3.

Generally, a line of $\mathfrak{M}$ is contained in at most one top, but it can be contained in many stars, cf. Table 4.

Lemma 1.2. A star and a top are disjoint, share a point or a line except an $\omega$-star and an $\alpha$-top that are disjoint or share a point. Two distinct tops are disjoint or share a point. Two distinct stars are disjoint or share a point, a line, a plane and so on up to a hyperplane. 


\subsection{Connectedness}

Let $U \in \operatorname{Sub}_{k}(V)$. The subspaces $\operatorname{Trc}(U)=W \cap U$ and $\operatorname{Ctr}(U)=W+U$ will be called a trace and cotrace of $U$ on $W$. The following is a quite technical, but important observation involving adjacency in $\mathbf{P}_{k}(V)$.

Lemma 1.3. For $i=1,2$, let $U_{i} \in \operatorname{Sub}_{k}(V), Z_{i}=\operatorname{Trc}\left(U_{i}\right) \in \operatorname{Sub}_{m}(W)$, and $Y_{i}=\operatorname{Ctr}\left(U_{i}\right)$. If $\operatorname{dim}\left(U_{1} \cap U_{2}\right)=k-1$ (i.e. $U_{1}, U_{2}$ are adjacent in $\mathbf{P}_{k}(V)$ ), then either $Z_{1}=Z_{2}$ and $Y_{1} / W, Y_{2} / W$ are adjacent in $\mathbf{P}_{k-m}(V / W)$ or $Y_{1}=Y_{2}$ and $Z_{1}, Z_{2}$ are adjacent in $\mathbf{P}_{m}(W)$.

Proof. Assume that $U_{1}, U_{2} \in \mathbf{p}(H, B)$, i.e. $H=U_{1} \cap U_{2}$ and $B=U_{1}+U_{2}$. Note, in view of Table 1 , that $\operatorname{dim}(\operatorname{Trc}(B))-\operatorname{dim}(\operatorname{Trc}(H))$ is 0 for $\alpha$-lines, 1 for affine lines and 2 for $\omega$-lines and respectively $\operatorname{dim}(\operatorname{Ctr}(B))-\operatorname{dim}(\operatorname{Ctr}(H))$ is $2,1,0$. For the argument it is enough to see that in the first and second cases we have $Z_{1}=Z_{2}$, while in the last one $Y_{1}=Y_{2}$.

From [1] we know that the complement of a hyperplane in a nondegenerate polar space is connected if the ambient polar space is of rank at least 3 . The complement of a hyperplane in a generalized quadrangle, i.e. in a polar space of rank 2, is also connected, which is proved in [4].

Lemma 1.4. Let $\mathfrak{A}=\langle S, \mathcal{L}\rangle$ be a polar space of rank at least 2 and let $X$ be its subspace which is not a hyperplane. The complement of $X$ in $\mathfrak{A}$, i.e. the fragment $\left\langle S \backslash X, \mathcal{L}_{S \backslash X}\right\rangle$ of $\mathfrak{A}$, is connected.

Proof. Take two distinct points $a, b \in S \backslash X$. As $X$ is not a hyperplane in $\mathfrak{A}$, there is a line $k \in \mathcal{L}$ which misses $X$, so $k \in \mathcal{L}_{S \backslash X}$. By the one-or-all axiom there are lines $l_{1} \in \mathcal{L}$ through $a$ and $l_{2} \in \mathcal{L}$ through $b$, such that both $l_{1}, l_{2}$ cross $k$. Let $l_{i} \cap X:=\left\{x_{i}\right\}$ and $l_{i}^{\prime}:=l_{i} \backslash\left\{x_{i}\right\}$ for $i=1,2$. Then, the path consisting of $l_{1}^{\prime}, k, l_{2}^{\prime}$ joins $a$ with $b$ in the complement of $X$ in $\mathfrak{A}$.

Lemma 1.5. Let $Z \in \operatorname{Sub}_{k-1}(W)$. The set

$$
\boldsymbol{S}(Z)=\left\{U \in \mathcal{F}_{k, k-1}(\mathrm{Q}, W): \operatorname{Trc}(U)=Z\right\}
$$

is a connected subspace of $\mathbf{A}_{k, k-1}(\mathrm{Q}, W)$.

Proof. If $\boldsymbol{S}(Z)$ is nonempty, then $Z$ is totally isotropic and the elements of $\boldsymbol{S}(Z)$ are $k$-subspaces contained in the segment $\left[Z, Z^{\perp}\right]$. In the induced polar space $V / Z$ they correspond to the set of 1 -subspaces which miss $W / Z$. So, this is a polar space with a subspace removed, which is connected by Lemma 1.4 or by $[1,4]$ in case $W$ is a hyperplane in $V$.

Lemma 1.6. If $\mathfrak{M}$ is connected then $m=w$ or $m<r_{W}$.

Proof. Assume that $\mathfrak{M}$ is connected. If all points of $\mathfrak{M}$ have a common trace, then $w=m$. Let $U_{1}, U_{2}$ be points of $\mathfrak{M}$ with $\operatorname{Trc}\left(U_{1}\right) \neq \operatorname{Trc}\left(U_{2}\right)$. Write $Z_{i}=$ 
$\operatorname{Trc}\left(U_{i}\right)$ for $i=1,2$. As $\mathfrak{M}$ is connected, there is a sequence of pairwise adjacent points joining $U_{1}$ and $U_{2}$. This means, by Lemma 1.3, that there is a path consisting of totally isotropic $m$-subspaces, joining $Z_{1}$ with $Z_{2}$ on $W$. So, there are at least two distinct, totally isotropic $m$-subspaces of $W$ that are adjacent, and thus $m<r_{W}$.

Recall that the complement of a subspace in a polar space is a semiaffine polar space (cf. [9]). In view of (12), from Lemmas 1.5 and 1.6 we get

Corollary 1.7. If $m=r_{W} \neq w$, then $\mathfrak{M}$ is disconnected. For $m=k-1$ its connected components are semiaffine polar spaces.

Example 1.8. Let us fix an integer $w$ with $2 l \leq w$. In $W=\mathbb{R}^{w}$ we define the following form

$$
\xi_{1}(x, y)=\sum_{i=1}^{w-l} x_{i} y_{i}-\sum_{i=1}^{l} x_{(w-l)+i} y_{(w-l)+i} ; \quad x, y \in \mathbb{R}^{w} .
$$

Then on $\mathbb{R}^{n-w}$ we consider the form $\xi_{2}$ defined by the condition

$$
\xi_{2}(x, y)=\sum_{i=1}^{(n-w)-(r-l)} x_{i} y_{i}-\sum_{i=1}^{r-l} x_{(n-w)-(r-l)+i} y_{(n-w)-(r-l)+i} ; \quad x, y \in \mathbb{R}^{n-w} .
$$

Finally we consider $V=\mathbb{R}^{n}=\mathbb{R}^{w} \oplus \mathbb{R}^{n-w}$ and we set

$$
\xi\left(\left[x^{\prime}, x^{\prime \prime}\right],\left[y^{\prime}, y^{\prime \prime}\right]\right)=\xi_{1}\left(x^{\prime}, y^{\prime}\right)+\xi_{2}\left(x^{\prime \prime}, y^{\prime \prime}\right) ; \quad x^{\prime}, y^{\prime} \in \mathbb{R}^{l}, x^{\prime \prime}, y^{\prime \prime} \in \mathbb{R}^{n-w} .
$$

Note that $l$ is the index of the form $\xi$ on $W$. For $l=m$, by Corollary 1.7, the polar spine space $\mathfrak{M}$ induced by this form on $V$ is disconnected.

\section{Maximal cliques}

Let $\mathfrak{M}$ be a polar spine space, i.e. $\mathfrak{M}=\mathbf{A}_{k, m}(\mathrm{Q}, W)$ for some integers $k, m$ and a fixed subspace $W$ of a vector space $V$.

Lemma 2.1. Flats and semibundles are $\boldsymbol{\pi}$-cliques.

Proof. It is evident that flats are $\boldsymbol{\pi}$-cliques. As the strong subspaces of $\mathfrak{M}$ are slit spaces any two lines in a semibundle are coplanar.

The proof of Proposition 2.2 in [8] remains true for general Grassmann spaces discussed in [7], for polar spine spaces in particular. Thus we have

Lemma 2.2. Every maximal $\boldsymbol{\pi}$-clique is either a flat or a semibundle. 
If $\boldsymbol{\Delta}_{\boldsymbol{\pi}}\left(L_{1}, L_{2}, L_{3}\right)$, then the lines $\overline{L_{1}}, \overline{L_{2}}, \overline{L_{3}}$ form a $\boldsymbol{\pi}$-clique which is either a tripod or a triangle not contained in two distinct maximal $\pi$-cliques. This means that they are not in a pencil. In view of Lemma 1.2 two stars can share a 3 -dimensional strong subspace if they are at least 4-dimensional. Moreover, if this is the case tripods contained in such stars do not satisfy $\boldsymbol{\Delta}_{\pi}$.

As on a plane the relation $\boldsymbol{\Delta}_{\pi}$ holds for every three lines we assume that every plane is contained in a 3-dimensional maximal strong subspace of $\mathfrak{M}$.

That means, every plane is contained in a star or a top of $\mathfrak{M}$. In Grassmann spaces and spine spaces, two distinct $\boldsymbol{\pi}$-cliques share at most one line. This is because two strong subspaces share at most a line. It is different in polar Grassmann spaces and polar spine spaces, where two star semibundles can have a lot more in common (comp. Lemma 1.2). Thus, Lemmas 2.5(i), 2.6(i), and consequently Proposition 2.7(i) from [8] cannot be directly repeated here.

Let $K$ be a semibundle which is a maximal $\pi$-clique. If $K$ is contained in two or more distinct stars then we need at least 3, up to $r-k+1$ lines (cf. Table 3) to span $K$. Now, the following is evident.

Lemma 2.3. For every maximal $\boldsymbol{\pi}$-clique $K$ there is an integer $s$ with $3 \leq s \leq$ $r-k+1$ and there are lines $L_{1}, L_{2}, \ldots, L_{s} \in K$ such that $\boldsymbol{\Delta}_{\pi}^{s}\left(L_{1}, L_{2}, \ldots, L_{s}\right)$ and $K=\left[L_{1}, L_{2}, \ldots, L_{s}\right]_{\pi} \in \mathcal{K}_{\pi}^{s}$. Consequently,

$$
\mathcal{K}_{\pi}=\bigcup\left\{\mathcal{K}_{\pi}^{s}: 3 \leq s \leq r-k+1\right\}
$$

and therefore the class $\mathcal{K}_{\pi}$ is definable in $\mathfrak{M}$.

Note that any 3-dimensional subspace that is contained in an $\alpha$-star can always be extended to another $\alpha$-star (cf. Theorem 4.4 in [5]) while this is not true for all $\omega$-stars. Hence, in contrast to $[8], \mathcal{K}_{\pi}^{3} \subsetneq \mathcal{K}_{\pi}$ and we get what follows.

Lemma 2.4. The set $\mathcal{K}_{\pi}^{3}$ consists of:

(i) flats,

(ii) top semibundles,

(iii) semibundles contained in 3-dimensional stars,

(iv) semibundles contained in w-stars which do not intersect any other star in a subspace of dimension 3.

In view of (7) the maximal $\boldsymbol{\pi}$-cliques (i)-(iv) are definable in $\langle\mathcal{L}, \boldsymbol{\pi}\rangle$.

As we are going to redefine $\mathfrak{M}$ using only top semibundles, we can restrict our attention to the class $\mathcal{K}_{\pi}^{3}$. 


\section{Proper semibundles}

We are going to identify points with vertices of proper semibundles, and we need proper pencils to do so. The reasoning presented in Section 3 of [8] shows how to define proper semibundles in spine spaces using lines and $\boldsymbol{\pi}$. Generally, this reasoning remains true for polar spine spaces. There are some differences though. So, we recall only the main steps without going into details, but we point out and explain the differences.

\subsection{Geometry on semibundles}

For three lines $L_{1}, L_{2}, L_{3} \in \mathcal{L}$ we make the following definition:

$$
\begin{gathered}
\mathbf{p}_{\boldsymbol{\pi}}\left(L_{1}, L_{2}, L_{3}\right) \quad \text { iff } \quad \text { there is } K \in \mathcal{K}_{\boldsymbol{\pi}}^{3} \quad \text { such that } \\
L_{1}, L_{2}, L_{3} \in K \quad \text { and } \neg \boldsymbol{\Delta}_{\boldsymbol{\pi}}\left(L_{1}, L_{2}, L_{3}\right) .
\end{gathered}
$$

This definition differs from its analogue in [8]. As it was mentioned, if $\boldsymbol{\Delta}_{\boldsymbol{\pi}}\left(L_{1}, L_{2}, L_{3}\right)$ then the lines $\overline{L_{1}}, \overline{L_{2}}, \overline{L_{3}}$ form a $\boldsymbol{\pi}$-clique, which is not a pencil or a tripod contained in a star, which shares a 3-dimensional subspace containing this tripod with another star. In view of Lemma 2.4, in order to rule out these tripods it suffices to assume that lines $L_{1}, L_{2}, L_{3}$ are in a set of the form (6), which is a member of the family $\mathcal{K}_{\boldsymbol{\pi}}$. Therefore, $\mathbf{p}_{\boldsymbol{\pi}}\left(L_{1}, L_{2}, L_{3}\right)$ means that $L_{1}, L_{2}, L_{3}$ form a proper pencil or a parallel pencil that is contained in a top or in a 3 -dimensional star or in an $\omega$-star which does not intersect any other star in a subspace of dimension 3 . Let us denote all these pencils by $\mathcal{P}_{\pi}$. As we see, unlike in [8], the family $\mathcal{P}_{\boldsymbol{\pi}}$ does not coincide with the family of all pencils. Nevertheless we get

Lemma 3.1. The family $\mathcal{P}_{\boldsymbol{\pi}}$ is definable in $\langle\mathcal{L}, \boldsymbol{\pi}\rangle$.

The next step is to distinguish and weed out parallel pencils in the family $\mathcal{P}_{\boldsymbol{\pi}}$. We have to do it, because proper semibundles contain no parallel pencils. The line of argumentation relies on the concept of coplanarity of two pencils, and splits up depending on whether the base plane of the pencil is affine or punctured (see Subsection 3.2 of [8]). Let $\mathcal{P}_{\|}$denote the set of all parallel pencils from the family $\mathcal{P}_{\boldsymbol{\pi}}$.

Lemma 3.2. The family $\mathcal{P}_{\|}$is definable in $\langle\mathcal{L}, \boldsymbol{\pi}\rangle$.

Set $\mathcal{P}:=\mathcal{P}_{\boldsymbol{\pi}} \backslash \mathcal{P}_{\|}$. According to Lemmas 3.1 and 3.2 in $\langle\mathcal{L}, \boldsymbol{\pi}\rangle$ we can also characterize the family $\mathcal{P}$.

The last step provides a distinction of some proper semibundles from flats.

Together with the proper pencils which it contains, every proper maximal $\boldsymbol{\pi}$-clique carries some geometry. The geometrical dimension of a flat is always 2 
whereas a proper semibundle $\mathrm{L}_{U}(X)$ has dimension one less than the dimension of $X$. So, under the assumption

stars or tops in $\mathfrak{M}$ are at least 4-dimensional projective or semiaffine spaces,

respectively, star semibundles or top semibundles are distinguishable from flats. However, if $\mathfrak{M}$ does not satisfy (16), then not all pencils of lines from the family $\mathcal{P}$ are definable in $\langle\mathcal{L}, \boldsymbol{\pi}\rangle$ and we need to use only those, denoted by $\mathcal{P}^{\prime}$, which are extendable to maximal $\boldsymbol{\pi}$-cliques (see Subsection 3.3 in [8]). Then, making the following definition

$$
\mathcal{B}^{\prime}:=\left\{K \in \mathcal{K}_{\pi}^{3}: \operatorname{dim}(K) \geq 3 \text { and there is } q \in \mathcal{P}^{\prime} \text { such that } q \subset K\right\}
$$

finally we get

Lemma 3.3. The family $\mathcal{B}^{\prime}$ defined in $\langle\mathcal{L}, \boldsymbol{\pi}\rangle$ coincides with the family of all proper top semibundles, the family of all proper semibundles contained in $\omega$ stars which do not intersect any other star in a subspace of dimension 3, or the union of these two families depending on whether tops, stars or all of them as projective or semiaffine spaces are at least 4-dimensional.

\subsection{Proper top semibundles}

The remainder of our reasoning is based strictly on proper top semibundles, which therefore have to be distinguished in the family $\mathcal{B}^{\prime}$. This means we have to assume that

all tops are at least 4-dimensional spaces,

which by Table 3 means that

$$
4 \leq k-m \text {. }
$$

By Lemma 3.3, if additionally all $\omega$-stars are at most 3 -dimensional spaces the proof is complete. In view of Table 3 this means that $\operatorname{dim}(W \cap Y)-m \leq 3$ for all $Y \in Q_{r}$, which is implied by $r_{W}-m \leq 3$. So, all $\omega$-stars are at most 3-dimensional under the condition

$$
r_{W} \leq m+3,
$$

which in some sense describes the polar geometry on $W$.

If (21) does not hold, the task is to distinguish top semibundles in $\mathcal{B}^{\prime}$. Following Lemma 3.3 it all comes to distinguishing tops and some at least 4 -dimensional $\omega$-stars, but the dimension criterion cannot be used to do it generally. The reason is that, in view of Table 3, the dimension of $\omega$-stars is not constant. Using intersections of tops and stars may be the next idea. But, in view of Lemma 1.2 and Table 4, this does not work either. 
Our method is based on the fact that a maximal strong subspace is either a projective or semiaffine space. This way we can distinguish $\omega$-tops from $\omega$ stars. We are left with $\alpha$-tops and $\omega$-stars. Looking at dimensions in Table 3 we have to assume that $\operatorname{dim}(W \cap Y) \neq k-2 m$ for all $Y \in \mathrm{Q}_{r}$, which means that there is no $(k-2 m)$-dimensional isotropic subspace in $W$, and is equivalent to

$$
r_{W} \leq k-2 m-1 \text {. }
$$

The problem may arise when there are lines that have no proper extensions. However, under assumption (20) this is not the case here. Indeed, if $r-k=1$ or $\operatorname{dim}(W \cap Y)-m=1$ for some $Y \in \mathrm{Q}_{r}$, there appear $\alpha$-lines or $\omega$-lines, respectively, that cannot be extended to stars, but they still have proper top extensions.

Summing up, proper top semibundles $\mathcal{B}$ are distinguishable in $\mathcal{B}^{\prime}$ if we assume

$$
\text { (20) and }((21) \text { or }(22))
$$

\section{Bundles}

On the family $\mathcal{B}$ of proper top semibundles we define

$$
\begin{gathered}
\Upsilon\left(K_{1}, K_{2}\right) \Longleftrightarrow\left(\exists L_{1}, L_{2} \in K_{1}\right)\left(\exists M_{1}, M_{2} \in K_{2}\right) \\
{\left[L_{1} \neq L_{2} \wedge L_{1} \pi M_{1} \wedge L_{2} \pi M_{2}\right] .}
\end{gathered}
$$

The next lemma, which is false for stars, shows that we need to know how to distinguish top semibundles.

Lemma 4.1. Let $X_{i}$ be a top and $K_{i}:=\mathrm{L}_{U_{i}}\left(X_{i}\right) \in \mathcal{B}, i=1,2$. If $\Upsilon\left(K_{1}, K_{2}\right)$ and $K_{1} \cap K_{2}=\emptyset$, then $U_{1}=U_{2}$.

Proof. By (23) there are lines $L_{1}, L_{2} \in K_{1}$ and $M_{1}, M_{2} \in K_{2}$, such that $L_{1} \neq L_{2}, L_{1} \pi M_{1}$ and $L_{2} \pi M_{2}$. Assume that $U_{1} \neq U_{2}$. Let $E_{i}$ be the plane spanned by $L_{i}, M_{i}, i=1,2$. We have $Z_{i} \in L_{i} \cap M_{i}$ which can be an improper point for $i=1,2$. Since $U_{1} \neq U_{2}$ and $K_{1} \cap K_{2}=\emptyset, Z_{i} \neq U_{i}$ for $i=1,2$. Note that $Z_{1}, Z_{2} \in \overline{X_{1}} \cap \overline{X_{2}}$. As $X_{1}, X_{2}$ are distinct tops, by Lemma 1.2 we get $Z_{1}=Z_{2}$. Then it follows that $L_{1}=L_{2}$, a contradiction. So $U_{1}=U_{2}$.

As in [8] we need to exclude punctured spaces here. Roughly, we do this because one affine line through a proper point is not enough in view of (23). The condition

$$
\text { no top is a punctured polar space, }
$$

is, by Table 3 , equivalent to

$$
k \neq m+1 \text {. }
$$


Note that (25) is implied by (20), and thus under the condition (20) no tops are punctured.

Lemma 4.2. Let $X_{i}=\left[\Theta, B_{i}\right]_{k}$ be a top, $B_{1} \perp B_{2}$ and $K_{i}:=\mathrm{L}_{U_{i}}\left(X_{i}\right) \in \mathcal{B}$, $i=1$, 2. If $U_{1}=U_{2}$, then $\Upsilon\left(K_{1}, K_{2}\right)$ and $K_{1} \cap K_{2}=\emptyset$.

Proof. Set $U=U_{1}=U_{2}$. Let us start with the case where $X_{1}$ is a projective space, i.e. an $\alpha$-top. Take a line $L_{1}=\mathbf{p}\left(H, B_{1}\right) \in K_{1}$, where $H \in \operatorname{Sub}_{k-1}(U)$ and $M_{1}=\mathbf{p}\left(H, B_{2}\right)$. Note that $M_{1} \in K_{2}$ and by Fact 1.1 we have $L_{1} \boldsymbol{\pi} M_{1}$. It is seen that $L_{1}$ was taken arbitrarily, so for any other line $L_{2}$ distinct from $L_{1}$ we can repeat our reasoning.

Now, consider the case where $X_{1}$ is a semi-affine space, i.e. an $\omega$-top. As it is not punctured we can take in $K_{1}$ two distinct affine lines $L_{1}, L_{2}$. Each of them can be extended to a semi-affine star which, according to Lemma 1.2, intersects $X_{2}$ in a line. That way we get lines $M_{1}, M_{2}$ with the property that $L_{i} \boldsymbol{\pi} M_{i}, i=1,2$ by Fact 1.1 .

Hence $\Upsilon\left(K_{1}, K_{2}\right)$. In view of Lemma 1.2 we have $K_{1} \cap K_{2}=\emptyset$.

In view of Corollary 1.7, it is not generally true that polar spine spaces are connected. However, some connected examples can be induced by the form $\xi$ defined in Example 1.8. Recall that $\mathfrak{M}=\mathbf{A}_{k, m}(\mathrm{Q}, W)$. From now on assume that

$$
\text { the polar spine space } \mathbf{A}_{k+1, m}(\mathrm{Q}, W) \text { is connected. }
$$

Let $\bar{\Upsilon}$ be the transitive closure of $\Upsilon$. Note that if $\mathfrak{M}$ is a polar spine space of linear complements (cf. [14]), that holds if $m=0$ and $w=n-k$, then all lines are affine and all strong subspaces are affine spaces, so there are no punctured spaces.

Lemma 4.3. Let $X_{i}$ be a top and $K_{i}:=\mathrm{L}_{U_{i}}\left(X_{i}\right) \in \mathcal{B}, i=1$, 2. If $U_{1}=U_{2}$, then $\bar{\Upsilon}\left(K_{1}, K_{2}\right)$ and $K_{1} \cap K_{2}=\emptyset$.

Proof. Let $X_{i}=\left[\Theta, B_{i}\right]_{k}$ for some $B_{1}, B_{2}$. The polar spine space $\mathbf{A}_{k+1, m}(\mathrm{Q}, W)$ is connected, so we can take a sequence $D_{0}, D_{1}, \ldots, D_{t}$ of its points such that $D_{0}=B_{1}$ and $D_{t}=B_{2}$ and $D_{i} \perp D_{i+1}, 0 \leq i<t$. Applying Lemma $4.2 t$ times for tops $\left[\Theta, D_{i}\right]_{k}$ and $\left[\Theta, D_{i+1}\right]_{k}, 0 \leq i<t$ we are through.

We need an equivalence relation to form the bundle of all lines through a given point. For proper top semibundles $K_{1}, K_{2} \in \mathcal{B}$ we write

$\Upsilon_{\emptyset}\left(K_{1}, K_{2}\right)$ iff $\Upsilon\left(K_{1}, K_{2}\right), \Upsilon\left(K_{2}, K_{1}\right)$, and either $K_{1} \cap K_{2}=\emptyset$ or $K_{1}=K_{2}$.

As with spine spaces, for $K \in \mathcal{B}$ we consider the set

$$
\Lambda_{\overline{\Upsilon_{\emptyset}}}(K):=\bigcup\left\{K^{\prime} \in \mathcal{B}: \overline{\Upsilon_{\emptyset}}\left(K, K^{\prime}\right)\right\}
$$

where $\overline{\Upsilon_{\emptyset}}$ is the transitive closure of $\Upsilon_{\emptyset}$. 
Lemma 4.4. Let $U$ be a point and $X$ be a top. If $U \in X$, then

$$
\Lambda_{\overline{\Upsilon_{\emptyset}}}\left(\mathrm{L}_{U}(X)\right)=\{L \in \mathcal{L}: U \in L\} .
$$

Proof. It suffices to repeat the reasoning presented in the proof of Lemma 4.4 in [8] applying Lemmas 4.1 and 4.3 .

Now, each point $U$ of $\mathfrak{M}$ can be identified with the bundle of all lines through that point, i.e. with $\Lambda_{\overline{\Upsilon_{\emptyset}}}(K)$ by Lemma 4.4 for some semibundle $K \in \mathcal{B}$ with vertex $U$. Gathering all requirements that we have imposed on $\mathfrak{M}$ throughout this section, the theorem we have proved is as follows.

Theorem 4.5. Let $\mathfrak{M}=\mathbf{A}_{k, m}(\mathrm{Q}, W)$ be a polar spine space satisfying (20) and one of (21) or (22). If the polar spine space $\mathbf{A}_{k+1, m}(\mathrm{Q}, W)$ is connected, then $\mathfrak{M}$ and the structure of its lines together with coplanarity relation $\langle\mathcal{L}, \boldsymbol{\pi}\rangle$ are definitionally equivalent.

\section{Collinearity in the structure of proper pencils}

With a geometrical structure, like $\mathfrak{M}$ in Sect. 1 , we frequently associate the point-line structure $\mathbf{P}(\mathfrak{M})$ whose points are the lines of $\mathfrak{M}$ and whose lines are proper pencils. Then the relation $\boldsymbol{\rho}$ defined by (4) is the binary collinearity of $\mathbf{P}(\mathfrak{M})$. Let us apply this procedure to $\mathfrak{M}=\mathbf{A}_{k, m}(V, W)$. In view of Theorem 4.5 , the relation $\boldsymbol{\rho}$ can be defined in $\langle\mathcal{L}, \boldsymbol{\pi}\rangle$. As we noted, $\boldsymbol{\rho} \subset \boldsymbol{\pi}$. So, each $\boldsymbol{\rho}$-clique is a $\boldsymbol{\pi}$-clique as well. It is relatively easy to prove, combining results of Sect. 2 and techniques usually used to determine cliques of binary collinearity in affine-like spaces (i.e. geometries with nonprojective planes, cf. [11] or [8]), that the following holds.

Proposition 5.1. A set $K$ is a maximal $\boldsymbol{\rho}$-clique iff one of the following holds

(i) $K$ is a proper semibundle $K=\mathrm{L}_{U}(X)$, where $X$ is a star or a top with $\operatorname{dim}(X) \geq 3$ and $U \in X$ is a point of $\mathfrak{M}$, or

(ii) there is a plane $E$ of $\mathfrak{M}$ such that $K$ consists of all the projective lines contained in $E$ and of a selector of the family $\mathcal{A}(E) / \|$ i.e. for each affine line $L$ contained in $E, K$ contains exactly one line parallel to $L$. So, $K$ is a subset of the flat $\mathrm{L}(E)$.

Let $K$ be a maximal $\rho$-clique. The clique $K$ contains a triple $L_{1}, L_{2}, L_{3}$ such that $\boldsymbol{\Delta}_{\boldsymbol{\rho}}\left(L_{1}, L_{2}, L_{3}\right)$ iff

(i) $K$ is a proper semibundle as in (i) of Proposition 5.1 and either

(i.1) $X$ is a top, or

(i.2) $X$ is a star which does not intersect any other star in a subspace of $\operatorname{dim}=3$.

In both cases $K=\left[L_{1}, L_{2}, L_{3}\right]_{\rho}$ and for any $L_{1}, L_{2} \in K$ such that $L_{1} \boldsymbol{\rho} L_{2}, L_{1} \neq L_{2}$ there is $L_{3} \in K$ such that $\boldsymbol{\Delta}_{\boldsymbol{\rho}}\left(L_{1}, L_{2}, L_{3}\right)$. 
(ii) $K$ is contained in a flat with base $E$ such that

(ii.1) $E$ is a projective plane; in this case $K=\left[L_{1}, L_{2}, L_{3}\right]_{\rho}$, or

(ii.2) $E$ is a punctured projective plane, so $E$ contains exactly one parallel pencil and in this case exactly one of $L_{1}, L_{2}, L_{3}$ satisfying $\boldsymbol{\Delta}_{\rho}\left(L_{1}, L_{2}, L_{3}\right)$ is affine (say $\left.L_{3}\right)$. Then $\left[L_{1}, L_{2}, L_{3}\right]_{\rho}$ consists of $L_{3}$ and the projective lines contained in $E$.

In case (ii.2) there is a triple $L_{1}, L_{2}, L_{3}$ of lines such that

$$
\boldsymbol{\Delta}_{\rho}\left(L_{1}, L_{2}, L_{3}\right) \wedge K=\left[L_{1}, L_{2}, L_{3}\right]_{\rho} \wedge \forall L_{3}^{\prime} \in K\left(\boldsymbol{\Delta}_{\rho}\left(L_{1}, L_{2}, L_{3}^{\prime}\right) \Longrightarrow L_{3}^{\prime}=L_{3}\right) .
$$

The (unique) line $L_{3}$ in $K$ that satisfies (29) is the unique affine line in $K$. In cases (i) and (ii.1) one cannot find any triple in $K$ that satisfies (29).

Moreover, via (ii.2) we can characterize punctured projective flats: if $K=$ $\left[L_{1}, L_{2}, L_{3}\right]_{\rho}$ is contained in a punctured projective flat $\mathrm{L}(E)$ and $(29)$ is valid, then

$$
\mathrm{L}(E)=K \cup\left\{L_{3}^{\prime}: \boldsymbol{\Delta}_{\rho}\left(L_{1}, L_{2}, L_{3}^{\prime}\right) \wedge\left|K \backslash\left[L_{1}, L_{2}, L_{3}^{\prime}\right]_{\rho}\right|=1\right\} .
$$

Let us write $\mathcal{F}_{\mathrm{s}}$ for the class of all punctured projective flats and $\mathcal{F}_{\mathrm{a}}$ for the class of affine flats. As above, we can define the class $\mathcal{F}_{\mathrm{s}}$.

Next, we begin the analysis which will enable us to define the class $\mathcal{F}_{\text {a. Let }} \boldsymbol{\rho}\left(L_{1}, L_{2}, L_{3}\right)$ holds for three pairwise distinct lines. Suppose that $\neg \boldsymbol{\Delta}_{\rho}\left(L_{1}, L_{2}, L_{3}\right)$, but also there are no lines $M_{1}, M_{2}, M_{3}$ with $L_{1}, L_{2}, L_{3} \subset$ $\left[M_{1}, M_{2}, M_{3}\right]_{\rho}, \boldsymbol{\Delta}_{\rho}\left(M_{1}, M_{2}, M_{3}\right)$. In particular, $L_{1}, L_{2}, L_{3}$ are in no flat with projective or punctured projective base. Consequently, either

(i) $L_{1}, L_{2}, L_{3}$ are in a flat with an affine base, or

(ii) $L_{1}, L_{2}, L_{3}$ are in a star semibundle whose base intersects another star in at least 3-space.

In case $(\mathrm{i})$, the formula

$$
M_{i}, M_{i}^{\prime} \in\left[L_{1}, L_{2}, L_{3}\right]_{\rho} \wedge M_{i} \not \boldsymbol{} M_{i}^{\prime}, i=1,2 \wedge M_{1} \boldsymbol{\rho} M_{2} \Longrightarrow M_{1}^{\prime} \boldsymbol{\rho} M_{2}^{\prime}
$$

is valid when $L_{1}, L_{2}, L_{3}$ are not in a pencil (observe, that the assumptions of (31) yield $M_{i} \| M_{i}^{\prime}$ ) and is false otherwise. In case (ii) the formula (31) is simply false.

Concluding: in terms of $\rho$ we can characterize triangles on affine flats as well and then in terms of $\boldsymbol{\rho}$ we can define the class $\mathcal{F}_{\mathrm{a}}$.

Now, note that the right hand side of (17) with $\boldsymbol{\pi}$ replaced by $\boldsymbol{\rho}$ defines pencils on projective planes and triples of projective lines in sets of the form (ii.2). Let us exclude from the so obtained class triples that are contained in an element of $\mathcal{F}_{\mathrm{s}}$; the obtained class of pencils is denoted by $\mathcal{P}^{\mathrm{p}}$. We need to 
define pencils $\mathcal{P}^{\mathrm{a}}$ with affine and pencils $\mathcal{P}^{\mathrm{s}}$ with punctured projective bases. To this aim we modify (17) as follows:

$$
\begin{gathered}
\mathbf{p}_{\rho}^{\mathrm{t}}\left(L_{1}, L_{2}, L_{3}\right) \quad \text { iff there are } K_{1} \in \mathcal{F}_{\mathrm{t}}, K_{2} \in \mathcal{K}_{\boldsymbol{\rho}} \text { such that } \\
L_{1}, L_{2}, L_{3} \in K_{1}, K_{2} \text { and } K_{2} \nsubseteq K_{1}
\end{gathered}
$$

for $\mathrm{t}=\mathrm{s}$, a. The formula (32) defines the class $\mathcal{P}^{\mathrm{t}}$ so the class $\mathcal{P}=\mathcal{P}^{\mathrm{p}} \cup$ $\mathcal{P}^{\mathrm{s}} \cup \mathcal{P}^{\mathrm{a}}$ of pencils is definable. Consequently, we can-as in Sects. 3-4, under suitable dimensional assumptions - distinguish in the class $\mathcal{K}_{\rho}^{3}$ the class of top semibundles (note that now the semibundles in question are already proper) and repeating the construction of the relation $\Upsilon$ we arrive to our second main

Theorem 5.2. Under the assumptions of Theorem 4.5 the four structures:

$$
\mathfrak{M}, \quad \mathbf{P}(\mathfrak{M}), \quad\langle\mathcal{L}, \boldsymbol{\pi}\rangle, \quad \text { and } \quad\langle\mathcal{L}, \boldsymbol{\rho}\rangle
$$

are pairwise definitionally equivalent.

Open Access. This article is licensed under a Creative Commons Attribution 4.0 International License, which permits use, sharing, adaptation, distribution and reproduction in any medium or format, as long as you give appropriate credit to the original author(s) and the source, provide a link to the Creative Commons licence, and indicate if changes were made. The images or other third party material in this article are included in the article's Creative Commons licence, unless indicated otherwise in a credit line to the material. If material is not included in the article's Creative Commons licence and your intended use is not permitted by statutory regulation or exceeds the permitted use, you will need to obtain permission directly from the copyright holder. To view a copy of this licence, visit http:// creativecommons.org/licenses/by/4.0/.

Publisher's Note Springer Nature remains neutral with regard to jurisdictional claims in published maps and institutional affiliations.

\section{Appendix}

See Tables 1, 2, 3 and 4 .

Each strong subspace $X$ of a spine space is a slit space, that is a projective space $\mathbf{P}$ with a subspace $\mathcal{H}$ removed. In the extremes $\mathcal{H}$ can be void, then $X$ is basically a projective space, or a hyperplane, then $X$ is an affine space.

TABle 1. Classification of lines in a spine space $\mathbf{A}_{k, m}(V, W)$

\begin{tabular}{lll}
\hline Class & Representative line $g=\mathbf{p}(H, B) \cap \mathcal{F}_{k, m}(W)$ & $g^{\infty}$ \\
\hline $\mathcal{A}_{k, m}(W)$ & $H \in \mathcal{F}_{k-1, m}(W), B \in \mathcal{F}_{k+1, m+1}(W)$ & $H+(B \cap W)$ \\
$\mathcal{L}_{k, m}^{\alpha}(W)$ & $H \in \mathcal{F}_{k-1, m}(W), B \in \mathcal{F}_{k+1, m}(W)$ & - \\
$\mathcal{L}_{k, m}^{\omega}(W)$ & $H \in \mathcal{F}_{k-1, m-1}(W), B \in \mathcal{F}_{k+1, m+1}(W)$ & - \\
\hline
\end{tabular}


TABle 2. Classification of stars and tops in a spine space $\mathbf{A}_{k, m}(V, W)$

\begin{tabular}{llll}
\hline Class & Representative subspace & $\operatorname{dim}(\mathbf{P})$ & $\operatorname{dim}(\mathcal{H})$ \\
\hline $\mathcal{S}_{k, m}^{\omega}(W)$ & {$[H, H+W]_{k}: H \in \mathcal{F}_{k-1, m-1}(W)$} & $\operatorname{dim}(W)-m$ & -1 \\
$\mathcal{S}_{k, m}^{\alpha}(W)$ & {$[H, V]_{k} \cap \mathcal{F}_{k, m}(W): H \in \mathcal{F}_{k-1, m}(W)$} & $\operatorname{dim}(V)-k$ & $\operatorname{dim}(W)-m-1$ \\
$\mathcal{T}_{k, m}^{\alpha}(W)$ & {$[B \cap W, B]_{k}: B \in \mathcal{F}_{k+1, m}(W)$} & $k-m$ & -1 \\
$\mathcal{T}_{k, m}^{\omega}(W)$ & {$[\Theta, B]_{k} \cap \mathcal{F}_{k, m}(W): B \in \mathcal{F}_{k+1, m+1}(W)$} & $k$ & $k-m-1$ \\
\hline
\end{tabular}

TABLE 3. Classification of stars and tops in a polar spine space $\mathbf{A}_{k, m}(\mathrm{Q}, W)$

\begin{tabular}{llc}
\hline & \multicolumn{2}{l}{ Representative subspace } \\
\cline { 2 - 2 } Class & $\operatorname{dim}(\mathbf{P})$ & $\operatorname{dim}(\mathcal{H})$ \\
\hline $\mathcal{S}_{k, m}^{\omega}(\mathrm{Q}, W)$ & {$[H,(H+W) \cap Y]_{k}: H \in \mathcal{F}_{k-1, m-1}(\mathrm{Q}, W), Y \in \mathrm{Q}_{r}, H \subset Y$} \\
& $\operatorname{dim}(W \cap Y)-m$ & -1 \\
$\mathcal{S}_{k, m}^{\alpha}(\mathrm{Q}, W)$ & {$[H, Y]_{k} \cap \mathcal{F}_{k, m}(\mathrm{Q}, W): H \in \mathcal{F}_{k-1, m}(\mathrm{Q}, W), Y \in \mathrm{Q}_{r}, H \subset Y$} \\
& $r-k$ & $\operatorname{dim}(W \cap Y)-m-1$ \\
$\mathcal{T}_{k, m}^{\alpha}(\mathrm{Q}, W)$ & {$[B \cap W, B]_{k}: B \in \mathcal{F}_{k+1, m}(\mathrm{Q}, W)$} & -1 \\
& $k-m$ & $k$ \\
$\mathcal{T}_{k, m}^{\omega}(\mathrm{Q}, W)$ & {$[\Theta, B]_{k} \cap \mathcal{F}_{k, m}(\mathrm{Q}, W): B \in \mathcal{F}_{k+1, m+1}(\mathrm{Q}, W)$} \\
& $k$ & $k-m-1$ \\
\hline
\end{tabular}

TABLE 4. Possible intersections of stars and tops in a polar spine space $\mathbf{A}_{k, m}(\mathrm{Q}, W)$

\begin{tabular}{lllll}
\hline & $\mathcal{S}_{k, m}^{\omega}(\mathrm{Q}, W)$ & $\mathcal{S}_{k, m}^{\alpha}(\mathrm{Q}, W)$ & $\mathcal{T}_{k, m}^{\alpha}(\mathrm{Q}, W)$ & $\mathcal{T}_{k, m}^{\omega}(\mathrm{Q}, W)$ \\
\hline $\mathcal{S}_{k, m}^{\omega}(\mathrm{Q}, W)$ & Any & $\emptyset$, point & $\emptyset$, point & $m=0 \rightarrow \emptyset$, \\
& & & point $m>0 \rightarrow$ \\
& & $\emptyset$, point, line \\
$\mathcal{S}_{k, m}^{\alpha}(\mathrm{Q}, W)$ & & Any & $\emptyset$, point, line & $\emptyset$, point, line \\
$\mathcal{T}_{k, m}^{\alpha}(\mathrm{Q}, W)$ & & & $\emptyset$, point, equal, & $\emptyset$, point \\
$\mathcal{T}_{k, m}^{\omega}(\mathrm{Q}, W)$ & & & $\emptyset$, point, equal \\
\hline
\end{tabular}




\section{References}

[1] Cohen, A.M., Shult, E.E.: Affine polar spaces. Geometriae Dedicata 35, 43-76 (1990)

[2] Cohen, M.A.: Point-line spaces related to buildings. In: Buekenhout, F. (ed.) Handbook of Incidence Geometry, pp. 647-737. North-Holland, Amsterdam (1995)

[3] Karzel, H., Pieper, I.: Bericht über geschlitzte Inzidenzgruppen. Jber. Deutsch. Math.Verein. 70, 70-114 (1970)

[4] Pambuccian, V.: Affine generalized quadrangles: an axiomatization. Geometriae Dedicata 84, 1-23 (2001)

[5] Pankov, M.: Grassmannians of Classical Buildings. Algebra and Discrete Mathematics, vol. 2. World Scientific, Hackensack (2010)

[6] Pankov, M., Prażmowski, K., Żynel, M.: Geometry of polar Grassmann spaces. Demonstr. Math. 39(3), 625-637 (2006)

[7] Petelczyc, K., Żynel, M.: Coplanarity of lines in projective and polar Grassmann spaces. Aequationes Math. 90(3), 607-623 (2016)

[8] Petelczyc, K., Żynel, M.: Geometry on the lines of spine spaces. Aequationes Math. 92(2), 385-400 (2018)

[9] Petelczyc, K., Żynel, M.: The complement of a subspace in a classical polar space. Ars Math. Contemp. 17(2), 447-454 (2019)

[10] Prażmowski, K.: On a construction of affine Grassmannians and spine spaces. J. Geom. 72, 172-187 (2001)

[11] Prażmowski, K., Prażmowska, M.: Grassmann spaces over hyperbolic and quasi hyperbolic spaces. Math. Pannonica 17(2), 195-220 (2006)

[12] Prażmowski, K., Żynel, M.: Automorphisms of spine spaces. Abh. Math. Sem. Univ. Hamb. 72, 59-77 (2002)

[13] Prażmowski, K., Żynel, M.: Affine geometry of spine spaces. Demonstratio Math. 36(4), 957-969 (2003)

[14] Prażmowski, K., Żynel, M.: Geometry of the structure of linear complements. J. Geom. 79, 177-189 (2004)

[15] Prażmowski, K., Żynel, M.: Extended parallelity in spine spaces and its geometry. J. Geom. 85(1-2), 110-137 (2006)

[16] Radziszewski, K.: Subspaces and parallelity in semiaffine partial linear spaces. Abh. Math. Sem. Univ. Hamb. 73, 131-144 (2003)

[17] Shoenfield, J.R.: Mathematical Logic. Addison-Wesley, Reading (1967)

[18] Żynel, M.: Complements of Grassmann substructures in projective Grassmannians. Aequationes Math. 88(1-2), 81-96 (2014)

Krzysztof Petelczyc, Krzysztof Prażmowski and Mariusz ŻYNEL

Faculty of Mathematics

University of Białystok

K. Ciołkowskiego $1 \mathrm{M}$

15-245 Białystok

Poland

e-mail: kryzpet@math.uwb.edu.pl

Krzysztof Prażmowski

e-mail: krzypraz@math.uwb.edu.pl

Mariusz ŻYNEL

e-mail: mariusz@math.uwb.edu.pl

Received: July 8, 2019

Revised: June 4, 2020 